\title{
Linearização do Sistema resultante da Discretização da Equação de Burgers
}

\author{
Tadasi Matsubara Jr Neyva M. Lopes Romeiro \\ Departamento de Matemática, CCE, UEL \\ Rod. Celso Garcia Cid. Pr 445 Km 380, 86.057 - 970 \\ Londrina-Pr \\ E-mail: jumatsubara@hotmail.com,nromeiro@uel.br
}

Gustavo T. Naozuka Matheus P. Vellone Saulo M. Mastelini

Departamento de Ciências da Computação, CCE, UEL

Rod. Celso Garcia Cid. Pr 445 Km 380, 86.057 - 970

Londrina-Pr

E-mail: gtnaozuka@gmail.com, matheusvellone@hotmail.com, saulo_mastelini@hotmail.com

\begin{abstract}
Resumo
Os métodos numéricos oferecem uma ferramenta indispensável na determinação de soluções aproximadas, uma vez que muitas das soluções analíticas encontradas na literatura envolvem simplificações e descartam as não linearidades presentes na equação. A ideia básica desses métodos é o processo de discretização, que reduz o problema contínuo, com um número infinito de variáveis, em um problema discreto com um número finito de variáveis, podendo ser resolvido computacionalmente. No caso do método de diferenças finitas (MDF), sua base é puramente matemática, pois pode ser desenvolvido a partir das aproximações por séries de Taylor, que são mais econômicas computacionalmente. Nesse trabalho o MDF é usado como uma abordagem alternativa para gerar soluções de equações diferenciais parciais (EDP's), em particular será utilizado para aproximar os termos temporal, convectivo e difusivo da equação de Burgers. A não linearidade do sistema de equações gerado a partir da aplicação do MDF nos termos da equação de Burgers será contornada utilizando uma técnica numérica [1].
\end{abstract}

\section{Introdução}

A equação 1D de Burgers é dada por

$$
\frac{\partial u}{\partial t}+\frac{1}{2} \frac{\partial u^{2}}{\partial x}=v \frac{\partial^{2} u}{\partial x^{2}}
$$

onde $v$ é o coeficiente de viscosidade, $u=u(x, t)$, sendo $x$ e $t$ as variáveis espacial e temporal, respectivamente. A equação (1.1) é considerada uma forma simplificada das equações de NavierStokes. Bateman em 1915 [2] resolve equações com características parecidas com (1.1) para problemas 1D, porém, esta equação foi plenamente proposta como um modelo matemático por Burgers em 1948 [3], quando concluiu sua forma como um modelo na teoria da turbulência. Sua natureza versátil permite a modelação de problemas tão distintos como os correspondentes a propagação de sinais acústicos, estudo da turbulência, ou formação de ondas de choque. Nesse 
trabalho estuda-se a aplicação do MDF para gerar soluções numéricas focalizando a obtenção de códigos que forneçam soluções de (1.1). Usa-se aproximações de diferença avançada no termo temporal, de diferença central ponderada no termo espacial de primeira ordem e de diferença central no termo espacial de segunda ordem. Os resultados numéricos obtidos a partir da discretização e implementação via o MDF serão comparados com os resultados analíticos, com o auxilio do software Scilab.

\section{Método de Diferenças Finitas}

A técnica de diferenças finitas, consiste em definir uma malha, sobre uma região de interesse e aproximar os termos da EDP em pontos genéricos de uma malha, dados por $\left(x_{i}, t_{j}\right)$, obtendo assim equações de diferenças. De uma forma geral, obtém-se uma aproximação da solução nos pontos da malha $\left(x_{i}, t_{j}\right)$, dividindo o intervalo $[0, \mathrm{~L}]$ da variável espacial $x$, em M partes iguais de comprimento $h$, resultando em $\mathrm{M}+1$ pontos do tipo $x_{i}=i h, i=1,2, \ldots$, sendo $h=\frac{L}{M}$. Similarmente, dividindo o intervalo $[0, \mathrm{~T}]$ da variável temporal $t$, em $\mathrm{N}$ partes iguais de comprimento $k$, tem-se os $\mathrm{N}+1$ pontos $t_{j}=j k, j=1,2, \ldots$, onde $k=\frac{T}{N}$.

\section{Método de Solução}

Usando aproximações de diferença avançada no termo temporal $\partial u / \partial t$, de diferença central ponderada no termo espacial de primeira ordem não linear $\partial^{2} u / \partial x$ e de diferença central no termo espacial de segunda ordem $\partial^{2} u / \partial x^{2}$, obtemos, respectivamente, no ponto $(i, j+1)$

$$
\begin{gathered}
\frac{\partial u}{\partial t} \cong \frac{u_{i, j+1}-u_{i, j}}{k} \\
\frac{\partial u^{2}}{\partial x} \cong \frac{1}{2 h}\left[\theta\left(u_{i+1, j+1}^{2}-u_{i-1, j+1}^{2}\right)+(1-\theta)\left(u_{i+1, j}^{2}-u_{i-1, j}^{2}\right)\right] \\
\frac{\partial^{2} u}{\partial x^{2}} \cong \frac{1}{h^{2}}\left[u_{i+1, j+1}-2 u_{i, j+1}+u_{i-1, j+1}\right] .
\end{gathered}
$$

Substituindo (3.1)-(3.3) em (1.1), temos

$$
\begin{aligned}
\frac{u_{i, j+1}-u_{i, j}}{k} & +\frac{1}{4 h}\left[\theta\left(u_{i+1, j+1}^{2}-u_{i-1, j+1}^{2}\right)+(1-\theta)\left(u_{i+1, j}^{2}-u_{i-1, j}^{2}\right)\right] \\
& =\frac{v}{h^{2}}\left[u_{i+1, j+1}-2 u_{i, j+1}+u_{i-1, j+1}\right]
\end{aligned}
$$

para $i=1 \ldots M-1$ e $j=0 \ldots N$, com erro de truncamento de ordem um em $k$ e de ordem dois em $h$, ou seja, $O(k)+O\left(h^{2}\right)$.

Considerando $\theta=0$ em (3.4) tem-se um sistema de equações lineares em $u_{i, j+1}$,

$$
\frac{u_{i, j+1}-u_{i, j}}{k}+\frac{1}{4 h}\left(u_{i+1, j}^{2}-u_{i-1, j}^{2}\right)=\frac{v}{h^{2}}\left[u_{i+1, j+1}-2 u_{i, j+1}+u_{i-1, j+1}\right] .
$$

Para $0<\theta \leq 1$ obtém um sistema de equações não-lineares para $u_{i, j+1}$, sendo necessário o uso de técnicas iterativas para obter a solução do sistema. Uma destas técnicas pode ser obtida definindo o operador de diferença central e o operador da média, por $2 \delta_{x} u_{i, j}=u_{i+1, j}-u_{i-1, j} \mathrm{e}$ $u_{i, j}=\frac{1}{2}\left(u_{i+1, j}+u_{i-1, j}\right)$, respectivamente, reduzindo, desta forma a equação (3.4) em 


$$
\frac{u_{i, j+1}-u_{i, j}}{k}+\frac{1}{4 h}\left[\theta \delta_{x}\left(u_{i, j+1}^{2}\right)+(1-\theta) \delta_{x}\left(u_{i, j}^{2}\right)\right]=\frac{v}{h^{2}}\left[u_{i+1, j+1}-2 u_{i, j+1}+u_{i-1, j+1}\right] .
$$

Da expansão de Taylor em $u_{i, j+1}^{2}$ no ponto $(i, j)$ obtemos,

$$
u_{i, j+1}^{2}=u_{i, j}^{2}+k \frac{\partial u_{i, j}^{2}}{\partial t}+\frac{k^{2}}{2 !} \frac{\partial^{2} u_{i, j}^{2}}{\partial t^{2}}+\ldots=u_{i, j}^{2}+2 k u_{i, j} \frac{\partial u_{i, j}}{\partial t}+\ldots
$$

usando $\frac{\partial u_{i, j}}{\partial t} \cong\left[\frac{u_{i, j+1}-u_{i, j}}{k}\right]$ em (3.7), obtemos

$$
u_{i, j+1}^{2} \cong u_{i, j}^{2}+2 u_{i, j}\left(u_{i, j+1}-u_{i, j}\right) \cong u_{i, j}^{2}+2 u_{i, j} W_{i}
$$

onde $W_{i}=u_{i, j+1}-u_{i, j}$. Substituindo (3.8) em (3.4) obtemos, após algumas manipulações algébricas o sistema

$$
\begin{aligned}
\frac{W_{i}}{k} & =\frac{v}{h^{2}}\left[u_{i+1, j+1}-2 u_{i, j+1}-u_{i-1, j+1}\right] \\
& -\frac{1}{4 h}\left[2 \theta u_{i+1, j} W_{i+1}+2 \theta u_{i-1, j} W_{i-1}+u_{i+1, j}^{2}-u_{i-1, j}^{2}\right]
\end{aligned}
$$

Considerando $r=\frac{k}{h^{2}}$ e rearranjando a equação (3.9) obtemos a equação,

$$
\begin{aligned}
\left(\theta r h u_{i-1, j}\right. & +2 r v) W_{i-1}-2(1+2 r v) W_{i}+\left(2 r v-\theta r h u_{i+1, j}\right) W_{i+1} \\
& =\frac{r h}{2}\left(u_{i+1, j}^{2}-u_{i-1, j}^{2}\right)-2 r v\left(u_{i-1, j}-2 u_{i, j}+u_{i+1, j}\right)
\end{aligned}
$$

que, para $i=1 \ldots M-1$ e $j$ fixo resulta em um sistema linear de equações da forma $A X=B$ para $W_{i}$, com $0 \leq \theta \leq 1$.

A matriz A dos coeficientes de (3.10) é uma matriz tridiagonal da forma

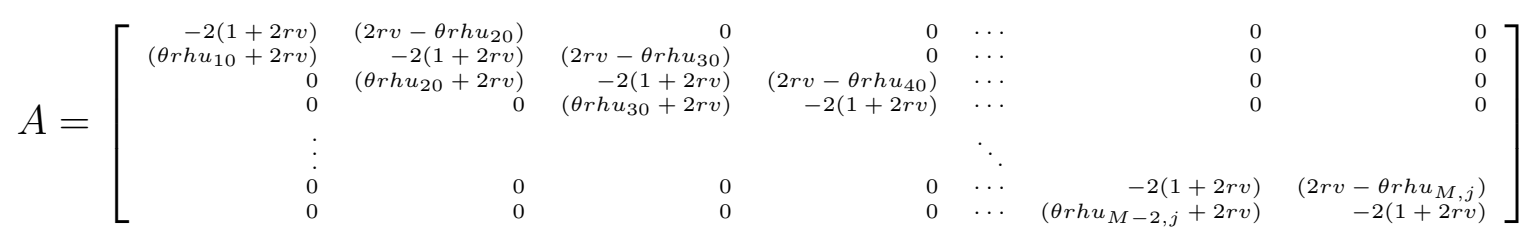

e os elementos do vetor B são

$$
\begin{gathered}
\frac{r h}{2}\left(u_{00}^{2}-u_{20}^{2}\right)+2 r v\left(u_{00}+2 u_{10}+u_{20}\right)-\left(\theta r h u_{00}+2 r v\right) W_{0} \\
\frac{r h}{2}\left(u_{10}^{2}-u_{30}^{2}\right)+2 r v\left(u_{10}+2 u_{20}+u_{30}\right) \\
\frac{r h}{2}\left(u_{20}^{2}-u_{40}^{2}\right)+2 r v\left(u_{20}+2 u_{30}+u_{40}\right) \\
\vdots \\
\frac{r h}{2}\left(u_{M, j}^{2}-u_{M-2, j}^{2}\right)+2 r v\left(u_{M-2, j}+2 u_{M-1, j}+u_{M, j}\right)-\left(2 r v-\theta r h u_{M, j}\right) W_{M} .
\end{gathered}
$$

O procedimento descrito, transforma o sistema não linear (3.4), gerado a partir da aplicação do MDF nos termos da equação de Burgers, em um sistema de equações lineares como apresentado em (3.10). 


\section{Resultados Numéricos}

Todos os resultados numéricos resultam da discretização e implementação da equação de Burgers a partir do método de diferenças finitas, utilizando a equação (3.10). Tais resultados encontram-se comparados com os resultados analíticos com o auxilio do software Scilab.

\subsection{Teste 1}

A equação 1D de Burgers definida em (1.1) com condição inicial $u(x, 0)=4 x(1-x)$ e condições de contornos $u(0, t)=0=u(1, t), t>0$, tem como solução analítica [4]

$$
u(x, t)=2 \pi v \frac{\sum_{n=1}^{\infty} a_{n} e^{-n^{2} \pi^{2} v t} n \operatorname{sen}(n \pi x)}{a_{0}+\sum_{n=1}^{\infty} a_{n} e^{-n^{2} \pi^{2} v t} \cos (n \pi x)}
$$

onde

$$
a_{0}=\int_{0}^{1} e^{-x^{2}(3 v)^{-1}(3-2 x)} d x
$$

$\mathrm{e}$

$$
a_{n}=2 \int_{0}^{1} e^{-x^{2}(3 v)^{-1}(3-2 x)} \cos (n \pi x) d x \quad n=1,2,3 \ldots
$$

Considerando o domínio $0 \leq x \leq 1$ apresentamos na Tabela 1 os resultados numéricos da equação de Burgers obtidos a partir da equação (3.10), para $\theta=0.1, \theta=0.5$ e $\theta=1.0$, comparados com a solução analítica dada em (4.1). Para o cálculo da solução analítica foi utilizado $n=20$, quanto aos demais parâmetros, utilizamos $v=0.1, h=0.05$ e $k=0.01$.

\begin{tabular}{|c|c|c|c|c|c|}
\hline \multirow[t]{2}{*}{$\mathrm{x}$} & \multirow[t]{2}{*}{$\mathrm{t}$} & \multicolumn{3}{|l|}{ S. Numérica } & \multirow[t]{2}{*}{ S. Analítica } \\
\hline & & $\theta=0.1$ & $\theta=0.5$ & $\theta=1.0$ & \\
\hline \multirow[t]{5}{*}{0.25} & 0.01 & 0.7276963 & 0.7277464 & 0.7278182 & 0.7274033 \\
\hline & 0.25 & 0.4064181 & 0.4061487 & 0.4058207 & 0.4053381 \\
\hline & 0.50 & 0.2778480 & 0.2776210 & 0.2773405 & 0.2774677 \\
\hline & 0.75 & 0.2098895 & 0.2097197 & 0.2095087 & 0.2097087 \\
\hline & 1.0 & 0.1657736 & 0.1656422 & 0.1654786 & 0.1655986 \\
\hline \multirow[t]{5}{*}{0.5} & 0.01 & 0.9920733 & 0.9923669 & 0.9927334 & 0.9916098 \\
\hline & 0.25 & 0.7230058 & 0.7242716 & 0.7259043 & 0.7186643 \\
\hline & 0.50 & 0.5177855 & 0.5181098 & 0.5185246 & 0.5153985 \\
\hline & 0.75 & 0.3891706 & 0.3892079 & 0.3892571 & 0.3876291 \\
\hline & 1.0 & 0.2997018 & 0.2996595 & 0.2996076 & 0.2983431 \\
\hline \multirow[t]{5}{*}{0.75} & 0.01 & 0.7563406 & 0.7563906 & 0.7564401 & 0.7567526 \\
\hline & 0.25 & 0.7516590 & 0.7549216 & 0.7591002 & 0.7486321 \\
\hline & 0.50 & 0.5771278 & 0.5793873 & 0.5822843 & 0.5719145 \\
\hline & 0.75 & 0.4164528 & 0.4175425 & 0.4189265 & 0.4119090 \\
\hline & 1.0 & 0.2994596 & 0.2999711 & 0.3006166 & 0.2958567 \\
\hline
\end{tabular}

Tabela 1: Comparações entre as soluções numéricas e analítica, considerando $v=0.1, h=0.05, k=0.01 \mathrm{e}$ diferentes valores para $\theta$.

Observamos, na Tabela 1 , que o valor de $\theta=0.1$ aproximou-se mais da solução exata, gerando erros relativos da ordem de $6.10^{-3}$. Devido aos resultados obtidos para $\theta=0.1$, apresentamos nas Figuras $1 \mathrm{a}-\mathrm{b}$ as superfícies das soluções numéricas e analíticas, e em 1c comparações dos resultados numéricos e analíticos considerando diferentes tempos. 

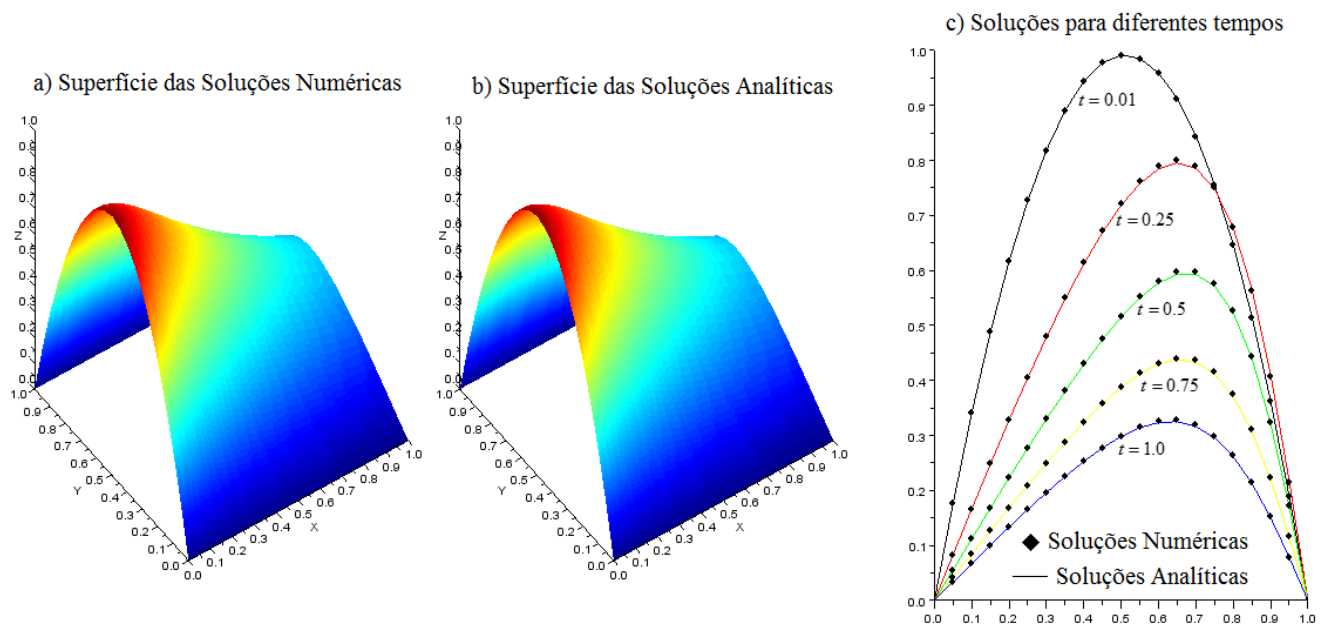

Figura 1: Soluções numéricas e analíticas do Teste 1

\subsection{Teste 2}

A equação 1D de Burgers definida em (1.1) com condição inicial dada por

$$
u(x, 0)=\frac{2 v \pi \operatorname{sen}(\pi x)}{a+\cos (\pi x)} \quad a>1,
$$

e condições de contornos $u(0, t)=0=u(1, t), t>0$, tem como solução analítica [5]

$$
u(x, t)=\frac{2 v \pi e^{\left(\pi^{2} v t\right)} \operatorname{sen}(\pi x)}{a+e^{\left(-\pi^{2} v t\right)} \cos (\pi x)} \quad a>1 .
$$

Considerando o domínio $0 \leq x \leq 1$ e $a=2$, apresentamos na Tabela 2 os resultados numéricos da equação de Burgers obtidos a partir de (3.10), para $\theta=0.1, \theta=0.5$ e $\theta=1.0$, $v=0.001, h=0.05, k=0.01$ e tempo final $t=1.0$, comparados com a solução analítica dada em (4.3).

Tabela 2: Comparações entre as soluções numéricas e analítica, considerando $v=0.001, h=0.02, k=0.01 \mathrm{e}$ diferentes valores para $\theta$.

\begin{tabular}{cccccc}
\hline $\mathrm{x}$ & $\mathrm{t}$ & S. Numérica & & & S. Analítica \\
\cline { 3 - 4 } & & $\theta=0.1$ & $\theta=0.5$ & $\theta=1.0$ & \\
\hline 0.25 & 0.01 & 0.0015760 & 0.0015760 & 0.0015760 & 0.0015763 \\
& 0.25 & 0.0015733 & 0.0015733 & 0.0015733 & 0.0015810 \\
& 0.50 & 0.0015704 & 0.0015704 & 0.0015704 & 0.0015860 \\
& 0.75 & 0.0015676 & 0.0015676 & 0.0015676 & 0.0015909 \\
& 1.0 & 0.0015647 & 0.0015547 & 0.0015547 & 0.0015959 \\
\hline 0.5 & 0.01 & 0.0031413 & 0.0031413 & 0.0031413 & 0.0031419 \\
& 0.25 & 0.0031339 & 0.0031339 & 0.0031339 & 0.0031494 \\
& 0.50 & 0.0031261 & 0.0031261 & 0.0031261 & 0.0031571 \\
& 0.75 & 0.0031184 & 0.0031184 & 0.0031184 & 0.0031649 \\
& 1.0 & 0.0031108 & 0.0031108 & 0.0031108 & 0.0031728 \\
\hline 0.75 & 0.01 & 0.0033834 & 0.0033834 & 0.0033834 & 0.0033841 \\
& 0.25 & 0.0033709 & 0.0033709 & 0.0033709 & 0.0033875 \\
& 0.50 & 0.0033579 & 0.0033579 & 0.0033579 & 0.0033911 \\
& 0.75 & 0.0033449 & 0.0033449 & 0.0033449 & 0.0033948 \\
& 1.0 & 0.0033320 & 0.0033320 & 0.0033320 & 0.0033985 \\
\hline
\end{tabular}


Observamos que para esse teste, independente do $\theta$, todas as soluções numéricas se aproximaram da solução exata, gerando erros relativos da ordem de $10^{-5}$. Apresentamos nas Figuras 2a-b as superfícies das soluções numéricas e analíticas, e em 2c comparações dos resultados numéricos e analíticos considerando o tempo final $t=1.0$ e $\theta=0.1$.
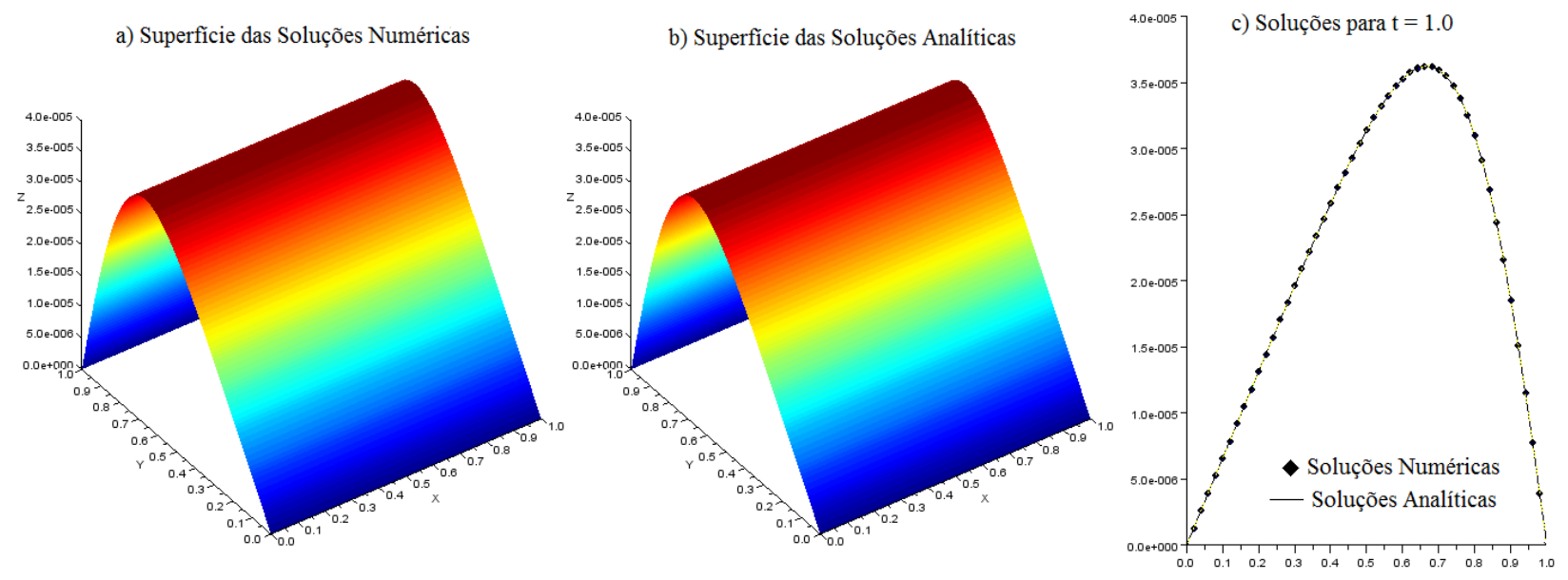

Figura 2: Soluções numéricas e analíticas do Teste 2.

\section{Conclusão}

Concluímos que a técnica utilizada para linearizar o sistema, gerado a partir da aplicação do MDF nos termos da equação de Burgers, mostrou-se eficiente quando comparando as soluções analíticas. Observamos que os resultados obtidos geraram erros de ordem $6.10^{-3}$ para o Teste 1 e de ordem $10^{-5}$ para o Teste 2 .

\section{Referências}

[1] KUTLUAY,S. e ESEN A. A linearized numerical scheme for Burgers-like equations. Applied Mathematics and Computation, Elsevier, 2003.

[2] BATEMAN, H, Some recent researches on the motion of fluids, Mon. Weather Rev. 43 (1915)163-170.

[3] BURGERS, J.M. A mathematical model illustrating the theory of turbulence, Adv. Appl. Mech. 1 (1948) 171-199.

[4] COLE, J.D., On a quasi-linear parabolic equations occurring in aerodynamics, Quart. Appl. Math., 9 (1951) 225-236.

[5] KUTLUAY, S., ESEN A., DAG, I., Numerical solutions of the Burgers equation by the least-squares quadratic B-spline finite element method, J. Comput. Appl. Math., 167 (2004) 21-33. 